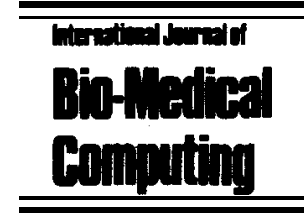

\title{
ANCOVA for nonparallel slopes: the Johnson-Neyman technique
}

\author{
Charles J. Kowalski ${ }^{a}$, Emet D. Schneiderman* ${ }^{\text {b }}$, Stephen M. Willis ${ }^{b}$ \\ ${ }^{a}$ Department of Biologic and Materials Sciences, Dental School, and The Center for Statistical Consultation and Research, \\ The University of Michigan, Ann Arbor, MI 48109 USA \\ bDepartment of Oral and Maxillofacial Surgery and Pharmacology, Baylor College of Dentistry, PO Box 660677, Dallas, \\ TX 75266-0677 USA
}

Received 10 March 1994; accepted 12 May 1994

\begin{abstract}
The Johnson-Neyman (JN) procedure, as originally formulated (Stat Res Mem, 1 (1936) 57-93), applies to a situation in which measurements on 1 dependent (response) variable, $\mathrm{X}$, and 2 independent (predictor) variables, $\mathrm{Z}$, and $\boldsymbol{Z}_{2}$, are available for the members of 2 groups. The expected value of $\mathrm{X}$ is assumed to be a linear function of $\mathrm{Z}$, and $\boldsymbol{Z}_{2}$, but not necessarily the same function for both groups. The JN technique is used to obtain a set of values for the $\mathrm{Z}$ variables for which one would reject, at a specified level of significance $\boldsymbol{\alpha}($ e.g., $\boldsymbol{\alpha}=\mathbf{0 . 0 5}$ ), the hypothesis that the 2 groups have the same expected X values. This set of values, or 'region of significance,' may then be plotted to obtain a convenient description of those values of $\mathrm{Z}$, and $Z_{2}$ for which the 2 groups differ. The technique can thus be described as a generalization of the analysis of covariance (ANCOVA) which does not make the assumption that the regression coefficients for the regression of $\mathrm{X}$ on the covariates, $\boldsymbol{Z}_{1}$ and $\boldsymbol{Z}_{2}$, are equal in the groups being compared. In this paper we describe, illustrate and make available a menu-driven PC program (TXJN2) implementing the JN procedure.
\end{abstract}

Keywords: Analysis of covariance; Nonparallel regressions; Region of significance; Three-dimensional graphics; PC program

\section{Introduction}

In a recent paper [1], we described and implemented a method for dealing with nonparallel regressions in the analysis of covariance (ANCOVA) where we had exactly 2 groups (TX = treatment, $\mathrm{C}=$ control), and pre- and post-TX measurements on each, the pre-TX value acting as

* Corresponding author. the (only) covariate. This was an application, in a special case, of the so-called Johnson-Neyman (JN) technique [2-5]. The JN technique has since been generalized to allow a larger number of covariates and, in this paper, we extend our discussion - and program - to accommodate 2 concomitant variables. The method and program are illustrated using 2 sets of data. The first has but a single covariate, and is used mainly to illustrate the computations, and to tie the present discussion 
and notation to [1]. In the second, 2 covariates are included. The technique is not limited to 2 covariates but, when more are present, the graphical part of the output -which is perhaps the most interesting from the practical standpoint - is difficult to implement. We begin with a description of the JN procedure. We maintain the basic notation established in [1]; in particular, $Z_{g i}$ denotes the pre-TX value for the $i^{\text {th }}$ individual $\left(i=1,2, \ldots n_{g}\right)$ in group $\mathrm{g}(\boldsymbol{g}=1,2)$, and $X_{g i}$ the corresponding post-TX value. Here, however, it will be convenient to use matrix notation in describing the computations. We follow [5] closely in our development.

\section{The Johnson-Neyman technique}

The JN procedure, as originally formulated in [2], applies in situations in which measurements on $1 \mathrm{X}$ variable and $2 \mathrm{Z}$ variables are available for each of the individuals comprising 2 groups. The JN technique is used to obtain a set of values of the $\mathrm{Z}$ variables for which one would reject the hypothesis that the 2 groups have the same expected $X$ value at a given level of significance (e.g., $\boldsymbol{\alpha}=0.05$ ). This set of $Z$ values is referred to as a 'region of significance'. This was illustrated for the special case of a single $\mathbf{Z}$ variable in [1].

We now consider the JN procedure when, say, $\mathrm{Q}$ covariates ( $Z$ variables) are available. These may be any measurements made at baseline, not only the premeasure matching the postmeasure, $\mathrm{X}$. It is assumed that the conditional distribution of $\boldsymbol{X}_{\boldsymbol{g} i}$ given $\mathbf{Z}_{\boldsymbol{g} \boldsymbol{i}}$ is normal (Gaussian) with the same variances in the 2 groups, and that

$$
E\left(X_{g i} \mid \mathbf{Z}_{g i}\right)=\beta_{0 g}+\beta_{g}^{\prime} \mathbf{Z}_{g i}
$$

We use boldface type to indicate vectors and/or matrices. In the above, $\mathbf{Z}_{g i}$ is $\mathrm{Q} \times 1$, containing the values of the covariates for a given individual; and $\boldsymbol{\beta}_{\boldsymbol{g}}$ is the Q x 1 vector of corresponding regression coefficients $\left(\boldsymbol{\beta}_{0 \mathrm{~g}}\right.$ is the 'intercept').

Letting 1 , denote the $n_{g} \times 1$ vector of 1 's, if we define

$$
\begin{aligned}
& \mathbf{Z}_{g}\left(Q \times n_{g}\right)=\left(\mathbf{Z}_{g 1}, \mathbf{Z}_{g 2}, \ldots \mathbf{Z}_{g n_{g}}\right) \\
& \mathbf{X}_{g}\left(n_{g \times 1}\right)=\left(X_{g 1}, X_{g 2}, \ldots, X_{g n_{g}}\right)^{\prime}
\end{aligned}
$$

$$
\begin{aligned}
& \overline{\mathbf{Z}}_{g}(Q \times 1)=\frac{1}{n_{g}} \mathbf{Z}_{g} \mathbf{1}_{g}, \overline{\mathbf{X}}_{g}=\frac{1}{n_{g}} \mathbf{X}_{g}^{\prime} \mathbf{1}_{g} \\
& \mathbf{C}_{g}(Q \times Q)=\mathbf{Z}_{g} Z_{g}^{\prime}-\frac{1}{n_{g}}\left(\mathbf{Z}_{g} \mathbf{1}_{g}\right)\left(\mathbf{Z}_{g} \mathbf{1}_{g}\right)^{\prime}
\end{aligned}
$$

and

$\mathrm{W}_{g}(Q \times 1)=\mathbf{Z}_{g} \mathbf{X}_{g}-\frac{1}{n_{g}}\left(\mathbf{Z}_{g} \mathbf{1}_{g}\right)\left(\mathbf{X}_{g}^{\prime} \mathbf{1}_{g}\right)$

then we can estimate

$\boldsymbol{\beta}_{\boldsymbol{g}}$ and $\boldsymbol{\beta}_{\mathbf{0 g}}$ by

$$
\mathbf{b}_{g}(Q \times 1)=\mathbf{C}_{g}^{-1} \mathbf{W}_{g}
$$

and

$$
b_{0 g}=\bar{X}_{g}-\mathbf{b}_{g}^{\prime} \overline{\mathbf{Z}}_{g}
$$

The error mean square will be written $s_{e}^{2}=S_{e}^{2} / f$ where

$$
S_{e}^{2}=\sum_{g=1}^{2}\left[\mathbf{X}_{g}^{\prime} \mathbf{X}_{g}-\frac{1}{n_{g}}\left(\mathbf{X}_{g}^{\prime} 1_{g}\right)^{2}-\mathbf{b}_{g}^{\prime} \mathbf{W}_{g}\right]
$$

and

$f=\sum_{g=1}^{2}\left(n_{g}-Q-1\right)$

These quantities are needed to estimate and judge the goodness-of-fit of the model. We turn now to constructing the region of significance. Let $\mathbf{Z}(Q \times 1)=\left[\begin{array}{lll}Z_{1}, Z_{2}, \ldots . & Z_{Q}\end{array}\right]^{\prime}$ denote a set of values of the $Z$ variables. Define

$$
\begin{aligned}
\Delta(\mathbf{Z}) & =\left(\beta_{01}+\beta_{1}^{\prime} \mathbf{Z}\right)-\left(\beta_{02}+\beta^{\prime}{ }_{2} \mathbf{Z}\right) \\
& =\left(\beta_{01}-\beta_{02}\right)+\left(\beta_{1}-\beta_{2}\right)^{\prime} \mathbf{Z}
\end{aligned}
$$

which is the true difference in expected values of $\mathrm{X}$ in the 2 groups at the 'point' $Z$. This can be estimated by

$$
D(\mathbf{Z})=\left(b_{01}-b_{02}\right)+\left(\mathbf{b}_{1}-\mathbf{b}_{2}\right)^{\prime} \mathbf{Z}
$$


We also define

$$
v(\mathbf{Z})=\sum_{g=1}^{2}\left[\frac{1}{n_{g}}+\left(\mathbf{Z}-\overline{\mathbf{Z}}_{g}\right)^{\prime} \mathbf{C}_{g}^{-1}\left(\mathbf{Z}-\overline{\mathbf{Z}}_{g}\right)\right]
$$

Then the JN 'region of significance' consists of the set of all points $Z$ such that

$$
\mathrm{R},(\mathrm{Z})=D^{2}(\mathrm{Z})-t_{1-\alpha / 2}^{2}(f) v(\mathrm{Z}) s_{e}^{2}>0
$$

where $t_{1-\alpha / 2}(f)$ is the $1-\alpha / 2$ percentile of the $t$ distribution with $f$ degrees of freedom. We use the notation $\mathbb{R}$ to denote the set of points for which Eq. 14 is satisfied. For any point $\boldsymbol{Z}$ in $\boldsymbol{R}$, one can reject $\mathrm{A}(\mathrm{Z})=0$ at level $\boldsymbol{\alpha}$. This is for any specified individual point in $\boldsymbol{R}$. We use the notation $\boldsymbol{R}_{\boldsymbol{M}}(\mathbf{Z})$ to represent the marginal (one-at-a-time) function defining the JN region of significance. Simultaneous inferences, for all points in another, somewhat smaller set $\boldsymbol{R}_{\mathrm{S}}$, can be obtained by using

$$
\begin{aligned}
R_{S}(\mathbf{Z})= & D^{2}(\mathbf{Z}) \\
& -(Q+1) F_{1-\alpha}(Q+1, f) v(\mathbf{Z}) s_{e}^{2}>0
\end{aligned}
$$

where $F_{1-\alpha}(Q+1, f)$ is the $1-\alpha$ percentile of the F-distribution with $Q+1$ and $f$ degrees of freedom.

It is perhaps more useful to express Eqs. 14 and 15 in terms of confidence intervals for $\Delta(Z)$. For a specified Z, Eq. 14 becomes

$$
D(Z) \approx \approx t_{1-\alpha / 2}(f)\left[v(Z) s_{e}^{2}\right]^{1 / 2}
$$

while the simultaneous interval is, from Eq. 15,

$$
D(Z) \pm\left[(Q+1) F_{1-\alpha}(Q+1, f) v(Z) s_{e}^{2}\right]^{1 / 2}
$$

It may be appropriate to consider the difference between $\boldsymbol{R}_{\boldsymbol{M}}(\mathbf{Z})$ and $\boldsymbol{R}_{\boldsymbol{S}}(\mathbf{Z})$ in more detail. Given a single, prespecified point $\mathrm{Z}$, one can compute $R_{M}(\mathbf{Z})$ at that point: if $R,,(Z)>0$, the expected values of $X$ in the 2 groups differ significantly for that given value of $Z$. It does not, however, follow from this that there is a significant difference between the 2 groups simultaneously for all points in the region where $\boldsymbol{R}_{\boldsymbol{M}}(\mathbf{Z})>0$. A statement of this latter type requires the use of simultaneous confidence bounds, and this is what is afforded us by $\boldsymbol{R}_{\boldsymbol{S}}(\mathbf{Z})$. Having determined $\boldsymbol{R}_{\boldsymbol{S}}=\left\{Z: \boldsymbol{R}_{\boldsymbol{S}}(\mathbf{Z})>0\right\}$, one can conclude that the expected values of $\mathrm{X}$ in the 2 groups differ significantly for all points, $Z$, in $\mathbf{R}_{\boldsymbol{S}}$. This extension of the JN procedure was developed by Potthoff [4] who may be consulted for more details and examples.

\section{JN vs. ANCOVA}

It is usual to test the hypothesis $\boldsymbol{H}: \boldsymbol{\beta}_{1}=\boldsymbol{\beta}_{\mathbf{2}}$ before applying JN. If this $H$ is not contradicted by the data, one might prefer to use ANCOVA since he/she will generally be rewarded with sharper tests and/or confidence intervals. On the other hand, the JN technique will be preferred by others since it does not depend on the (albeit testable) assumption that $\boldsymbol{\beta}_{1}=\boldsymbol{\beta}_{2}$. In our program, we test $\boldsymbol{H}$, and perform the ANCOVA for those who wish to follow the common strategy of testing first and deciding later. To perform the test, we compute

$$
\mathrm{b}=\left(\mathbf{C}_{1}+\mathbf{C}_{2}\right)^{-1}\left(\mathbf{W}_{1}+\mathbf{W}_{2}\right)
$$

and

$$
\begin{aligned}
S_{e^{*}}^{2}= & \sum_{g=1}^{2}\left[\mathbf{X}_{g}^{\prime} \mathbf{X}_{g}-\frac{1}{n_{g}}\left(\mathbf{X}_{g}^{\prime} \mathbf{1}_{g}\right)^{2}\right] \\
& -\mathbf{b}^{\prime}\left(\mathbf{W}_{1}+\mathbf{W}_{2}\right)
\end{aligned}
$$

which is the error sum of squares (cf Eq. 9 ) under the model with $\boldsymbol{\beta}_{1}=\boldsymbol{\beta}_{2}$. Then

$$
F=\frac{\left(S_{e}^{2}-S_{e}^{2}\right) / Q}{S_{e}^{2} / f}
$$

may be compared with the $F\left(Q_{2}\right)$ distribution to test $\boldsymbol{\beta}_{1}=\boldsymbol{\beta}_{2}$. The $(1-\boldsymbol{\alpha}) \times 100 \%$ confidence interval for $\beta_{01}-\beta_{02}$ when $\beta_{1}=\beta_{2}$ is

$$
\overline{\mathrm{X}}_{1}-\overline{\mathrm{X}}_{2}-\mathbf{b}^{\prime}\left(\overline{\mathrm{Z}}_{1}-\overline{\mathrm{Z}}_{2}\right) \pm t^{*}\left[\frac{1}{n_{1}}+\frac{1}{n_{2}}\right.
$$




$$
\begin{aligned}
& \left.+\left(\overline{\mathbf{X}}_{1}-\overline{\mathbf{X}}_{2}\right)^{\prime}\left(\mathbf{C}_{1}+\mathbf{C}_{2}\right)^{-1}\left(\overline{\mathbf{X}}_{1}-\overline{\mathbf{X}}_{2}\right)\right] \\
& \times\left[\frac{S_{e^{*}}^{2}}{n_{1}+n_{2}-Q-2}\right]^{1 / 2}
\end{aligned}
$$

where $t^{*}$ denotes the value $t_{1-\alpha / 2}\left(n_{1}+n_{2}-\mathrm{Q}-\right.$ 2).

\section{Examples}

We first consider data originally collected by Rikkers et al. [6] and later used by Brogan and Kutner [7] to compare and contrast some of the analyses which are often used in two-group, pretest/posttest situations. The data were gathered as part of a prospective randomized surgical trial in which cirrhotic patients were allocated to either a nonselective shunt (standard operation or 'control') or to a selective shunt (new operation). The dependent variable is the maximal rate of urea synthesis (MRUS), which is a measure of kidney function: poor liver function is associated with low MRUS values. The purpose of the analysis is to test for a difference between the treatments, using the pretreatment measurement as the (single) covariate. The data, as assembled into an ASCII file for input into our program, are shown below:
15148
13555
16660
14035
13936
14643
15246
14254
23416
24036
23416
23618
23832
23214
24420
25043
26045
26367
25036

\section{4}

24332

The 3 columns comprising the data set are, respectively, the group indicator variable, and the preand post-measurements. The $\mathrm{T}$ group is indicated by a ' 1 ' in column 1 ; the $C$ group by a ' 2 ' in column 1. Upon issuing the command gsruni txjn2, and giving the name and location of the ASCII file, the user is prompted for the confidence coefficient to be used in constructing the confidence intervals (it is set at 0.95 for this example).

For this data set, we have

$$
\begin{aligned}
& \mathbf{Z}_{1}=[51,35,66,40,39,46,52,42] \\
& \mathbf{Z}_{2}=[34,40,34,36,38,32,44,50,60,63,50,42,43] \\
& \mathbf{X}^{\prime}{ }_{1}=[48,55,60,35,36,43,46,54] \\
& \mathbf{X}^{\prime}{ }_{2}=[16,36,16,18,32,14,20,43,45,67,36,34,32]
\end{aligned}
$$

The first piece of output is a plot of the individual changes as shown in Fig. 1. These are color coded (on the screen) for group membership. This plot may be useful in describing the overallinterindividual patterns of change, group differences in change patterns, and in identifying outliers. The numbers on the far right of the inset refer to the case numbers of subjects in the data set. Next we plot the mean changes as shown in Fig. 2. It is clear from Fig. 2 that TX group (\#1) is maintaining their MRUS values, while those who received the standard operation (\#2) are deteriorating.

The descriptive statistics for the 2 groups are printed in the form:

NUMBER OF SUBJECTS: 21

TREATMENT GROUP:

$\mathrm{N} 1=8$

$$
\begin{aligned}
& \text { ZBAR } 1=46.3750 \mathrm{SD}=9.8697 \mathrm{VAR}=97.4107 \\
& \text { XBAR } 1=42.1250 \mathrm{SD}=8.9831 \mathrm{VAR}=80.6964 \\
& \text { DBAR } 1=0.7500 \mathrm{SD}=9.7358 \mathrm{VAR}=94.7857
\end{aligned}
$$

$\mathrm{RZX}=0.4699$

$\mathrm{RZD}=-0.5802$ 


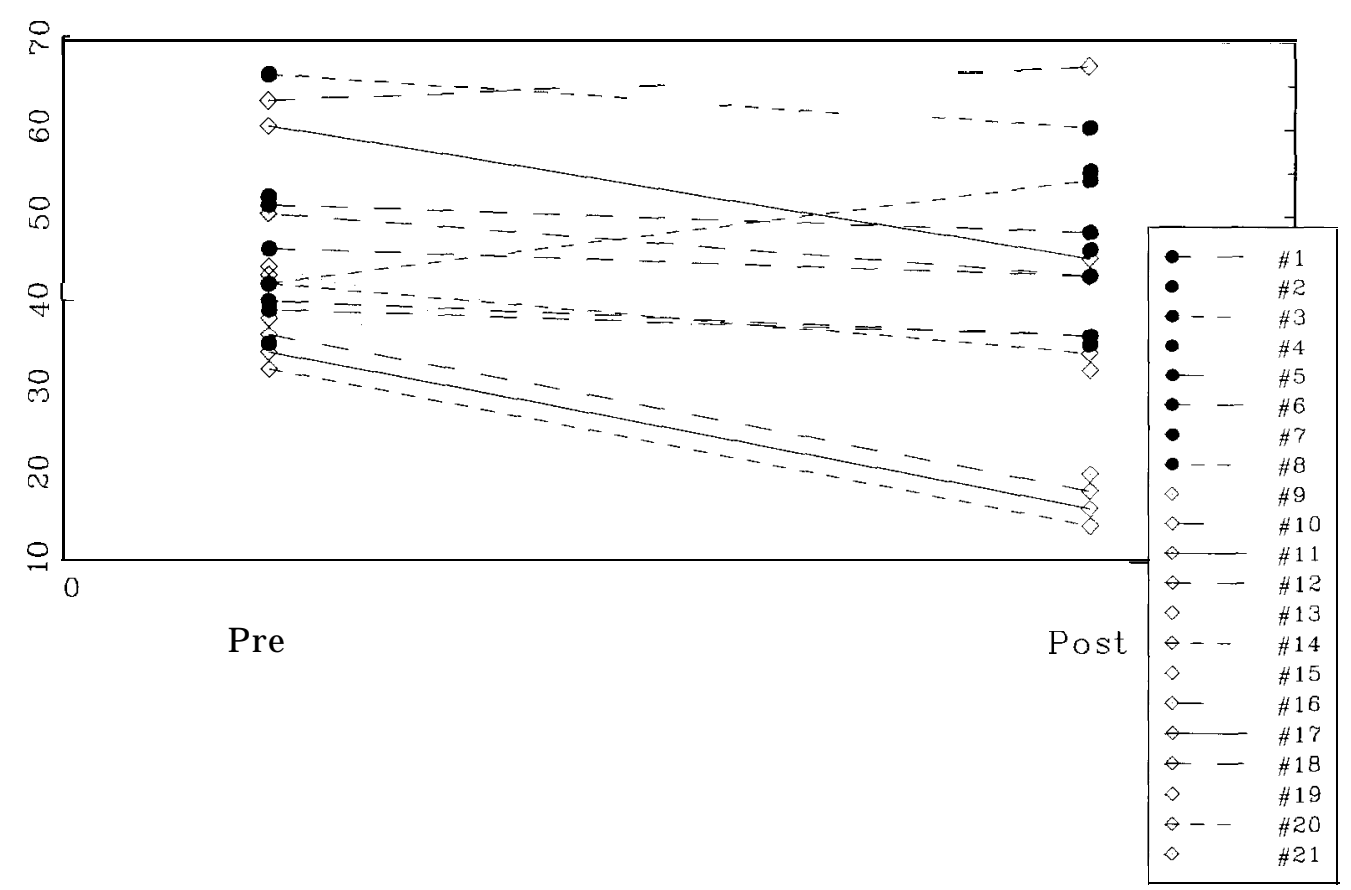

Fig. I. Pre- and post-individual MRUS values. Numbers in the inset refer to the sequential order of subjects in the data set.

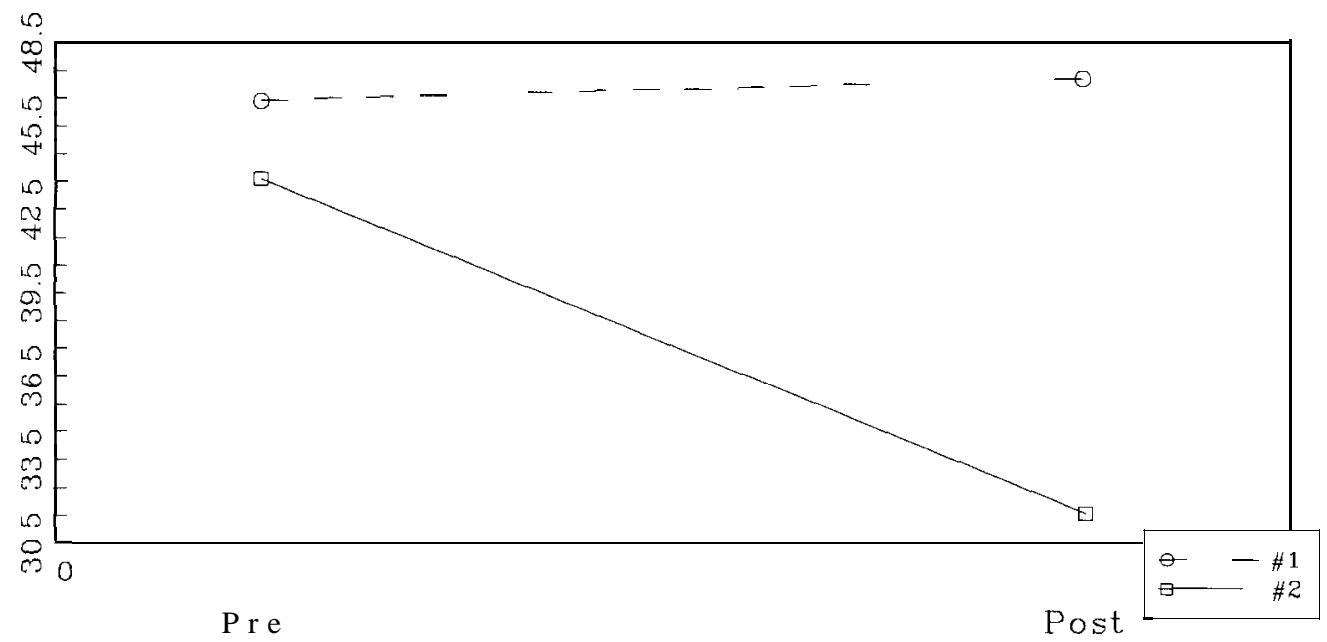

Fig. 2. Pre- and post-mean MRUS values in the 2 groups. \#1 is the treatment group; \#2 the control. 
CONTROL GROUP:

$\mathrm{N} 2=13$

$\mathrm{ZBAR} 2=43.5385 \mathrm{SD}=9.7947 \mathrm{VAR}=95.9359$

$\mathrm{XBAR} 2=31.4615 \mathrm{SD}=15.0644$

$$
\mathrm{VAR}=226.9359
$$

$\mathrm{DBAR} 2=-12.0769 \mathrm{SD}=7.6317$

$$
\mathrm{VAR}=58.2436
$$

$\mathrm{RZX}=0.8967$

$\mathrm{RZD}=0.4867$

We next compute

$C_{1}=Z_{1} Z_{1}^{\prime}-\frac{1}{8}\left(Z_{1} \mathbf{1}_{1}\right)\left(Z_{1} \mathbf{1}_{1}\right)^{\prime}=681.875$

$C_{2}=Z_{2} Z_{2}^{\prime}-\frac{1}{1_{1}^{2} J}\left(Z_{2} 1_{2}\right)\left(Z_{2} 1_{2}\right)^{\prime}=1151.2308$

$\mathbf{W}_{1}=\mathbf{Z}_{1} \mathbf{X}_{1}-\frac{1}{8}\left(\mathbf{Z}_{1} \mathbf{1}_{1}\right)\left(\mathbf{X}^{\prime}{ }_{1} \mathbf{1}_{1}\right)=291.625$
$\mathbf{W}_{2}=\mathbf{Z}_{2} \mathbf{X}_{2}-\frac{1}{13}\left(\mathbf{Z}_{2} \mathbf{1}_{2}\right)\left(\mathbf{X}_{2}^{\prime} \mathbf{1}_{2}\right)=1587.7692$

Then

$\mathbf{b}_{1}=\mathbf{C}_{1}^{-1} \mathbf{W}_{1}=0.4277$

$\mathbf{b}_{01}=\overline{\mathbf{X}_{1}}-\mathbf{b}_{1}^{\prime} \mathbf{Z}_{1}=27.2913$

$\mathbf{b}_{2}=\mathbf{C}_{2}^{-1} \mathbf{W}_{2}=1.3792$

$\mathbf{b}_{02}=\overline{\mathbf{X}}_{2}-\mathbf{b}^{\prime}{ }_{2} \overline{\mathbf{Z}}_{2}=-28.5864$

and

$S_{e}^{2}=\mathbf{X}_{1}^{\prime} \mathbf{X}_{1}-\frac{1}{8}\left(\mathbf{X}_{\mathrm{I}}^{\prime} \mathbf{1}_{1}\right)-\mathbf{b}_{1}^{\prime} \mathbf{W}_{1}+\mathbf{X}_{2}^{\prime} \mathbf{X}_{2}$

$$
-\frac{1}{13}\left(X_{2}^{\prime} 1_{2}\right)-b_{2}^{\prime} W_{2}=973.5434
$$

$f=(8-2)+(13-2)=17$

$s_{\mathrm{e}}^{2}=S_{\mathrm{e}}^{2} / f=57.2673$

Our program next plots separately fitted regressions of the postmeasures on the premeasures in the 2 groups, as shown in Fig. 3. As mentioned

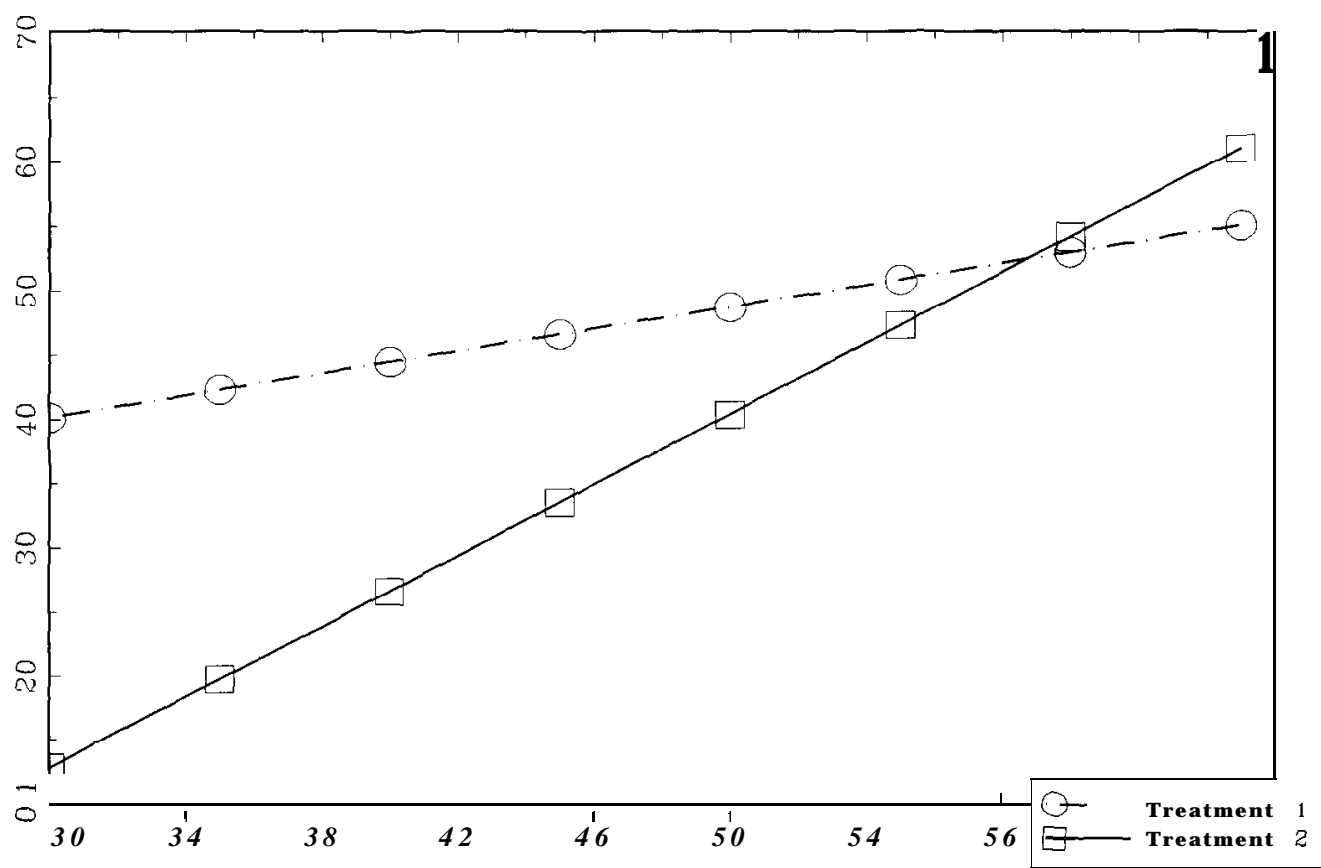

Fig. 3. Separately fitted regressions of postmeasures on initial MRUS values. One must assume that these are parallel to proceed with ANCOVA. 
earlier, the ANCOVA assumes these are parallel, contrary to what Fig. 3 seems to indicate.

To carry out the formal test for equality of slopes, we compute

$$
\begin{gathered}
\mathrm{b}=\left(\mathrm{C},+\mathbf{C}_{2}\right)^{-1}\left(\mathbf{W}_{1}+\mathrm{W},\right)=1.0253 \\
S_{e^{2}}=\mathbf{X}_{1}^{\prime} \mathbf{X}_{1}-\frac{1}{8}\left(\mathbf{X}_{1}^{\prime} \mathbf{1}_{1}\right)+\mathbf{X}_{2}^{\prime} \mathbf{X}_{2}-\frac{1}{13}\left(\mathbf{X}_{2}^{\prime} \mathbf{1}_{2}\right) \\
\quad-\mathbf{b}^{\prime}\left(\mathbf{W}_{1}+\mathrm{W},\right)=1361.2542
\end{gathered}
$$

and

$$
\mathbf{F}=\frac{S_{e^{*}}^{2}-S_{e}^{2}}{S_{e}^{2} / f}=6.77
$$

Our program summarizes the above information by printing

$$
\begin{aligned}
& \text { ESTIMATES OF PARAMETERS } \\
& \mathbf{B} 1=0.4277 \\
& \mathrm{~B} 2=1.3792 \\
& \text { B01 }=27.2913 \\
& \mathrm{~B} 02=-28.5864 \\
& \mathrm{~B}=1.0253 \\
& \mathrm{SSEF}=973.5434 \\
& \text { DFF }=17 \\
& \mathrm{MSEF}=57.2673 \\
& \text { SSER }=1361.2542 \\
& \text { F OBSERVED }=6.77
\end{aligned}
$$$$
\text { P VALUE }=0.0186
$$

Most of this output is self-explanatory. Some terminology which may be useful in connecting the above development to the general linear model is: SSEF is the error sum-of-squares for the full model $\left(S_{\mathrm{e}}^{2}\right)$; DFF is the number of degrees of freedom for the full model $(f)$; MSEF is mean square error for the full model $\left(s_{\mathrm{e}}^{2}\right)$; and SSER is the error sum-of-squares for the reduced model $\left(S_{\mathrm{e}^{*}}^{2}\right)$. This is consistent with the terminology employed in $[8,9]$.
In any event, since $F_{0.95}(1,17)=4.45$, we reject equality of slopes at the $5 \%$ level of significance (our program computes the $\mathbf{P}$ value for this hypothesis, viz., $\mathbf{P}=0.0186$ ). This indicates that the difference between the adjusted mean differences for the groups depends on $Z$. When $\beta_{1} \neq \beta_{2}$, the difference between the groups may be significant for certain values of $\boldsymbol{Z}$; not significant for others. And this is what the JN procedure is designed to accomplish - to determine those values of $\boldsymbol{Z}$ for which significant differences exist. Thus if one rejects $\boldsymbol{H}: \boldsymbol{\beta}_{1}=\boldsymbol{\beta}_{2}$ or, simply, does not choose to make this assumption, the user will continue with the JN analysis which constitutes the subsequent output from our program.

The value of $\mathrm{D}(\mathrm{Z})$ as given in Eq. 12 and the lower and upper limits for the marginal and simultaneous confidence intervals defined in Eqs. 16 and 17, respectively for several values of $Z$ are shown below and plotted in Fig. 4. The user has control of the values of $\mathrm{Z}$ for which the confidence intervals are to be computed, and which values of $\mathrm{Z}$ will be included in the plots. He/she is prompted for the initial and final values of $\mathrm{Z}$ and the increment between them. In the example below, we use $30(5) 65$, i.e., we begin at $Z=30$ and work up to $Z=65$ in steps of 5 . (The value $Z=51$ was computed separately to make another point.) In Fig. 4, the confidence intervals shown are the simultaneous intervals.

\begin{tabular}{lcccccc}
\hline & & \multicolumn{2}{l}{ Marginal } & & \multicolumn{2}{l}{ Simultaneous } \\
\cline { 3 - 4 } \cline { 6 - 7 }$Z$ & $D(Z)$ & Lower & Upper & & Lower & Upper \\
\hline 30 & 21.33 & 13.46 & 41.20 & & 9.15 & 44.95 \\
35 & 22.51 & 11.80 & 33.35 & & 8.89 & 36.26 \\
40 & 17.82 & 9.48 & 26.15 & & 1.23 & 28.40 \\
45 & 13.06 & 5.80 & 20.32 & & 3.84 & 22.28 \\
50 & 8.30 & 0.20 & 16.40 & & -1.99 & 18.59 \\
51 & 7.35 & -1.12 & 15.82 & & -3.41 & 18.12 \\
55 & 3.54 & -6.81 & 13.96 & & -9.68 & 16.77 \\
60 & -1.21 & -14.66 & 12.24 & & -18.3 & 15.87 \\
65 & -5.97 & -22.80 & 10.86 & & -27.4 & 15.41 \\
\hline
\end{tabular}

If 0 (zero) is in the confidence interval corresponding to a given value of $\boldsymbol{Z}$, there is no significant difference between the groups at that value of $\mathrm{Z}$. 
It is seen from the numerical results and/or Fig. 4 that the region of significance consists of those values of $Z$, roughly, for which $Z<50$. One can also get some feeling for the differences in the widths of the marginal and simultaneous confidence intervals from the above results. The simultaneous intervals are considerably wider. While $Z=50$ is in the marginal region of significance, it is not in the simultaneous region of significance. The largest integral value of $\mathrm{Z}$ which is in the simultaneous region of significance is $Z=48$ (cf Fig. 4). At that point, $D(Z)=10.21$ and the $95 \%$ simultaneous interval is $(0.625,19.79)$.

Our next example is a continuation of the first. We add a second covariate (age) to illustrate the output in this situation. $\mathbf{X}_{\mathbf{1}}$ and $\mathbf{X}_{\mathbf{2}}$ are as before, but now

$\mathbf{z}_{1}=\left[\begin{array}{llllllll}60 & 93 & 65 & 48 & 69 & 40 & 60 & 68\end{array}\right]$

and

$\mathbf{Z}_{\mathbf{2}}=\left[\begin{array}{lllllllllllll}34 & 40 & 34 & 36 & 38 & 32 & 44 & 50 & 60 & 63 & 50 & 42 & 43 \\ 64 & 62 & 61 & 58 & 60 & 69 & 65 & 72 & 75 & 70 & 69 & 59 & 62\end{array}\right]$

where the second rows of $\mathbf{Z}_{1}$ and $\mathbf{Z}_{\mathbf{2}}$ are the (fictitious) ages of the subjects.

Without showing the intermediate computations, the descriptive statistics in this case are now given in the form:

\section{NUMBER OF SUBJECTS: 21}

TREATMENT GROUP:

$\mathrm{N} 1=8$

ZBAR11 $=46.3750$ SD1 $1=9.8697$

VAR $11=97.4107$

ZBAR12 $=62.2500$ SD12 $=3.2404$

VAR12 $=10.5000$

XBAR $1=42.1250 \mathrm{SD}=8.9831 \mathrm{VAR}=80.6964$

DBAR $1=0.7500 \mathrm{SD}=9.7358 \mathrm{VAR}=94.7857$
RZX11 $=0.4699$
RZX12 $=0.6466$
RZD11 $=-0.5802$
RZD12 $=0.3962$

CONTROL GROUP:

$\mathrm{N} 2=13$

ZBAR21 $=43.5385$ SD21 $=9.7947$

VAR21 $=95.9359$

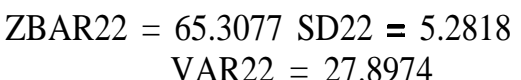

$\mathrm{XBAR} 2=31.4615 \mathrm{SD}=15.0644$

$\mathrm{VAR}=226.9359$

DBAR2 $=-12.0769 \mathrm{SD}=7.6317$

$\mathrm{VAR}=58.2436$

$\mathbf{R Z X 2 1}=0.8967$
$\mathrm{RZX} 22=0.4547$

RZD21 $=0.4867$

RZD22 $=0.0544$

These are, of course, much the same as before except now we have a second covariate and the output is labeled to reflect this fact. For example, RZD22 is the correlation between $\boldsymbol{D}$ and $\boldsymbol{Z} 2$ in group 2, i.e., the first 2 signifies group membership, the second the second covariate. It is seen that the average age for subjects in the second (control) group is somewhat higher than that for group one $(\mathrm{ZBAR} 12=62.25, \mathrm{ZBAR} 22=65.3)$.

Next we print

\section{ESTIMATES OF PARAMETERS}

$\mathbf{B 1}=0.32401 .5974$ 


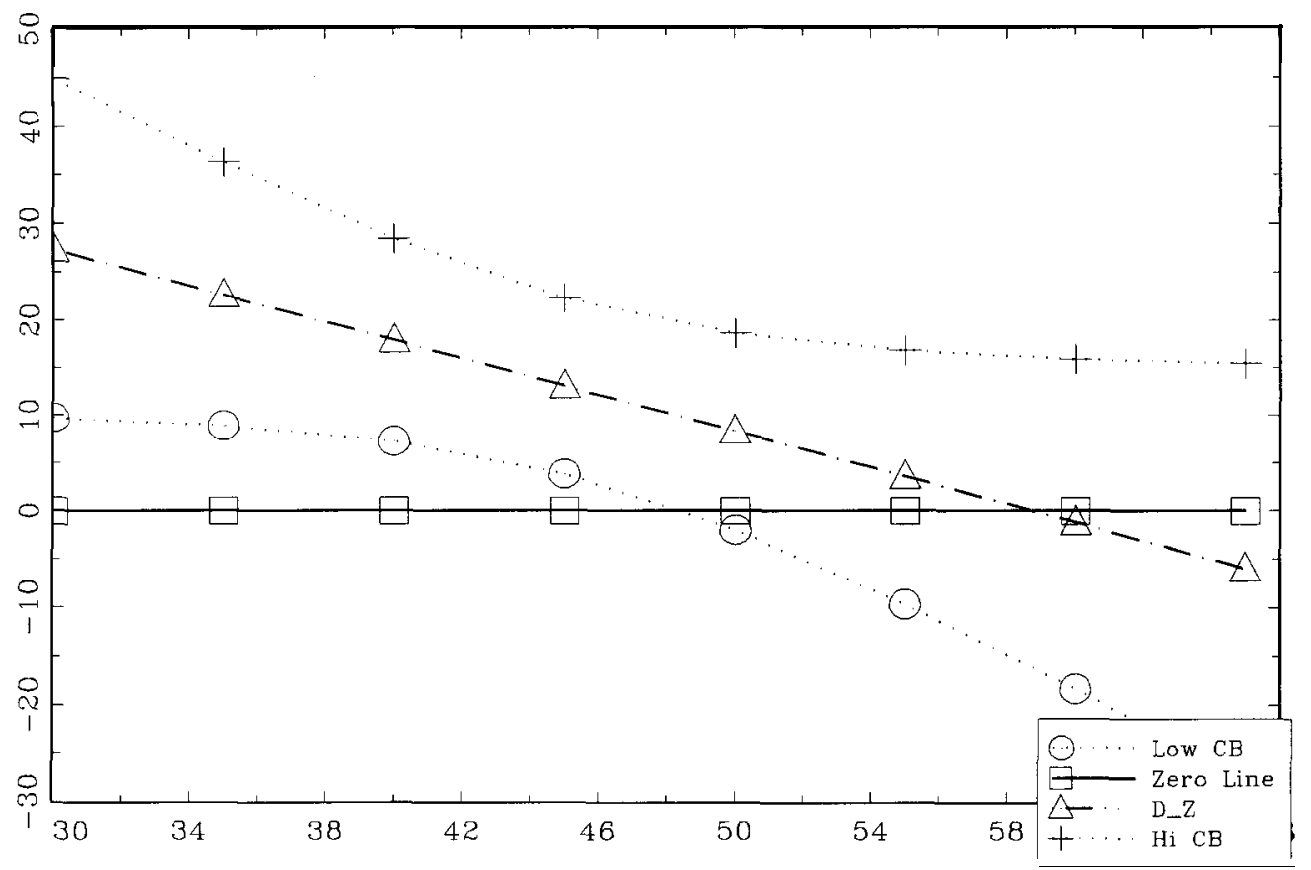

Fig. 4. Region of significance for the MRUS data. Values of $\mathrm{Z}$ for which the zero line is not within the confidence bands constitute this region, i.e., values of $\mathrm{Z}<48$.

$\mathrm{B} 2=1.6181-0.6745$

$\mathrm{B} 01=-67.3423$

$\mathrm{B} 02=5.0598$

$\mathrm{B}=0.9402$

$\mathrm{SSEF}=706.7$

$\mathrm{DFF}=15$

$\mathrm{MSEF}=47.12$

SSER $=1326$

F OBSERVED $=6.5708$

$\mathrm{P}$ VALUE $=0.0089$

It is seen that we reject the equality of slopes hypothesis. This would ordinarily prompt users to not use the ANCOVA and to proceed to the JN technique. Nevertheless, regardless of the outcome of this test for equality of slopes, at this point in our program we print the confidence interval (Eq.

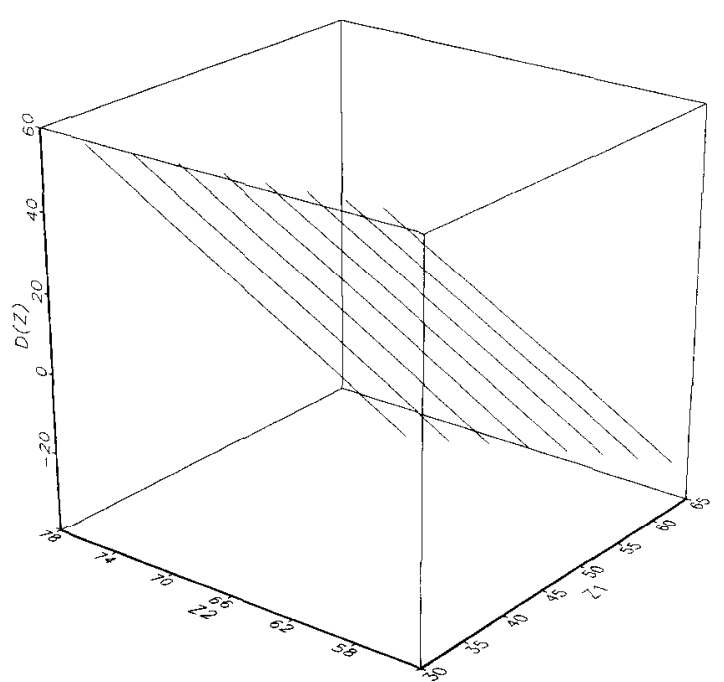

Fig. 5. $\mathrm{D}(\mathrm{Z})$ for various values of initial MRUS value and age. 
21) for $\boldsymbol{\beta}_{01}-\boldsymbol{\beta}_{02}$ which is computed under the assumption that $\boldsymbol{\beta}_{1}=\boldsymbol{\beta}_{2}$. In the context of our example, the program prints

Confidence interval for $\mathbf{B} \mathbf{0 1}-\mathrm{B} 02$ assuming $\mathbf{B} 1=\mathrm{B} 2$

\begin{tabular}{ll}
\hline Lower limit & Upper limit \\
\hline 4.662 & 23.441 \\
\hline
\end{tabular}

This confidence interval corresponds to what one would get from the routine use of the ordinary ANCOVA. If the data do not contradict the hypothesis $\boldsymbol{\beta}_{\mathbf{1}}=\boldsymbol{\beta}_{2}$, the user may wish to stop at this juncture, using the above confidence interval to assess possible differences between the groups. If zero is not in this interval, the groups differ; if it is, they do not. In this example, there is a significant difference between $\boldsymbol{\beta}_{01}$ and $\boldsymbol{\beta}_{\mathbf{0 2}}$ since zero is not contained in $(4.662,23.441)$.

Continuing with the JN procedure, the user next specifies ranges of values of $\boldsymbol{Z}_{1}$ and $\boldsymbol{Z}_{2}$, and the increments to be used in evaluating and plotting $\mathbf{D}(\mathbf{Z})$ and $\mathbf{R}(\mathbf{Z})$, as in our earlier example. Choosing $\boldsymbol{Z}_{\mathbf{1}}$ to go from 30 to 65 in steps of 5 ; and $\boldsymbol{Z}_{\mathbf{2}}$ to go from 55 to 75 in steps of 5 , we get 2 sets of numerical results. For each combination of values of $\boldsymbol{Z}_{1}$ and $\boldsymbol{Z}_{2}$, the first set consists of the values of $\mathbf{D}(\boldsymbol{Z})$ and the lower and upper limits for both the marginal and simultaneous confidence intervals for $A(Z)$. The first 5 lines (rounded) of this output is shown below:

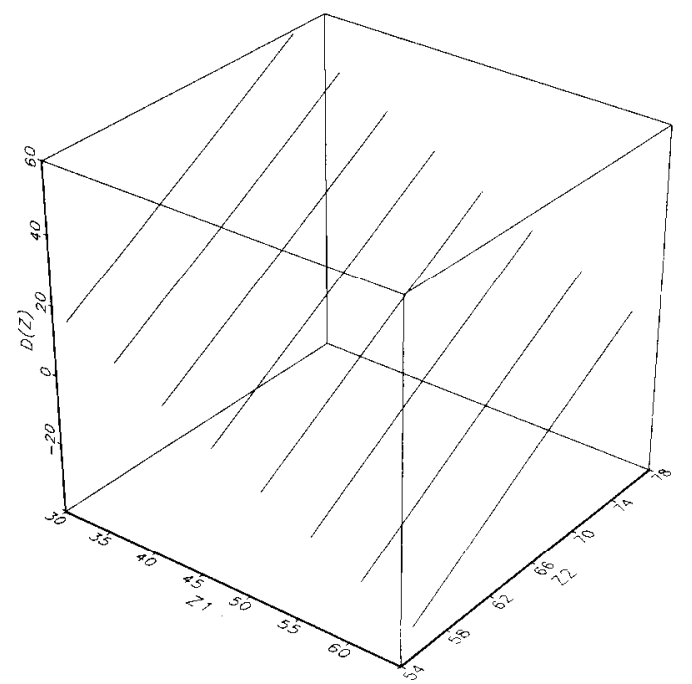

Fig. 6. $D(Z)$ for various values of initial MRUS value and age after rotation of the axes (cf Fig. 5).

\begin{tabular}{llll}
\hline $\mathbf{Z I}$ & $\mathbf{Z 2}$ & Marginal R(Z) & Simultaneous R(Z) \\
\hline 30 & 55 & -123.3 & -488.2 \\
30 & 60 & 463.4 & 269.0 \\
30 & 65 & 1100 & 833.2 \\
30 & $\mathbf{7 0}$ & 1787 & 1204 \\
30 & $\mathbf{7 5}$ & 2525 & 1382 \\
\hline
\end{tabular}

Various plots of $\mathbf{D}(\mathbf{Z})$ and $\mathbf{R}(\mathbf{Z})$ vs. $\boldsymbol{Z}_{1}$ and $\boldsymbol{Z}_{\mathbf{2}}$ are shown in Figs. 5- 14. The plots are designed to show those values of $Z_{1}$ and $Z_{2}$ such that $\mathrm{D}(Z)$ and $\mathbf{R}(\boldsymbol{Z})$ are greater than zero. When $\mathrm{D}(Z)>0$, the difference between the TX and control groups

\begin{tabular}{|c|c|c|c|c|c|c|}
\hline \multirow[t]{2}{*}{$\mathrm{ZI}$} & \multirow[t]{2}{*}{$\mathrm{Z} 2$} & \multirow[t]{2}{*}{$\mathrm{D}(\mathrm{Z})$} & \multirow{2}{*}{$\frac{\text { Marginal }}{\text { Lower limit }}$} & \multirow{2}{*}{$\frac{\text { Marginal }}{\text { Upper limit }}$} & \multirow{2}{*}{$\frac{\text { Simultaneous }}{\text { Lower limit }}$} & \multirow{2}{*}{$\begin{array}{l}\text { Simultaneous } \\
\text { Upper limit }\end{array}$} \\
\hline & & & & & & \\
\hline 30 & 55 & 13.73 & -3.93 & 31.39 & -12.28 & 39.14 \\
\hline 30 & 60 & 25.09 & 12.20 & 31.98 & 6.10 & 44.08 \\
\hline 30 & 65 & 36.45 & 21.34 & 51.56 & 14.19 & 58.71 \\
\hline 30 & 70 & 47.81 & 25.49 & 70.13 & 14.92 & 80.70 \\
\hline 30 & 75 & 59.17 & 27.92 & 90.42 & 13.14 & 105.2 \\
\hline
\end{tabular}

In the second set of numerical output, again for each combination of $\boldsymbol{Z}_{1}$ and $\boldsymbol{Z}_{2}$, we give the values of $\boldsymbol{R}_{\boldsymbol{M}}(\mathbf{Z})$ and $\boldsymbol{R}_{\boldsymbol{S}}(\mathbf{Z})$, viz., 


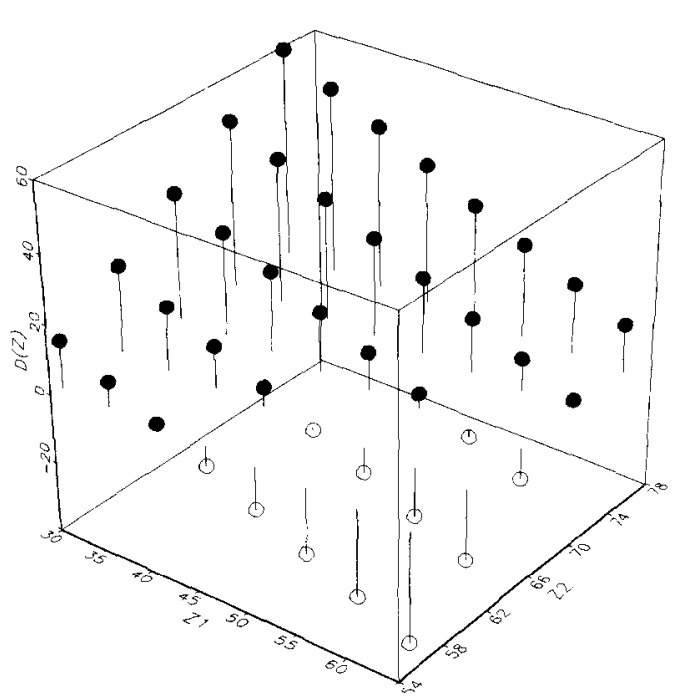

Fig. 7. $D(Z)$ for various values of initial MRUS value and age.

is positive; when $\mathbf{R}(\mathbf{Z})>\mathbf{0}$, this difference is significant. These plots are produced sequentially in the program and, while any given plot is on the screen, the user may print it by pressing the space bar and selecting one of the printing options shown.

In Fig. 5, the plane $\mathbf{D}(\mathbf{Z})$ is shown at the selected values of $\mathbf{Z}$. Note the orientation of the $Z_{1}$ and $Z_{2}$

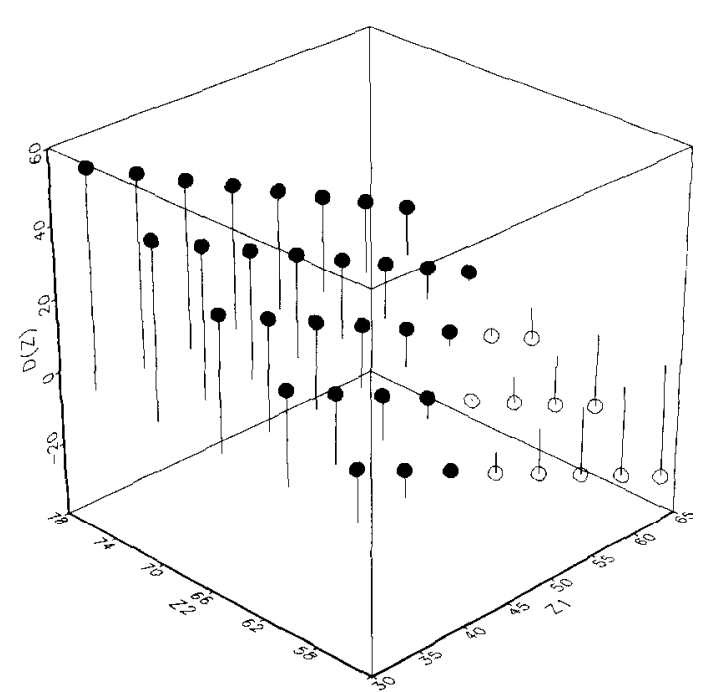

Fig. 8. $D(Z)$ for various values of initial MRUS value and age after rotation of theaxes (cf Fig. 7).

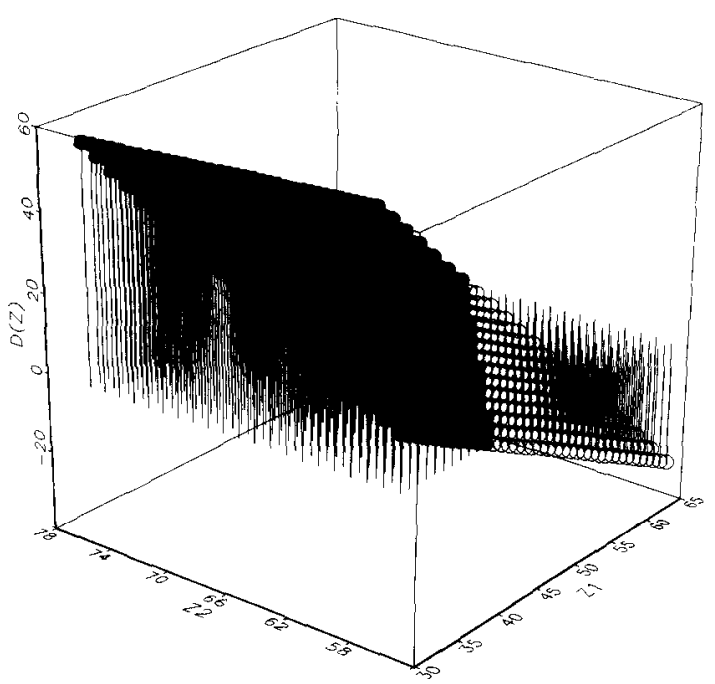

Fig. 9. $D(Z)$ for various values of initial MRUS value and age when more $Z$ values are selected for plotting (cf Fig. 8).

axes. While the plot is on the screen, the user may choose to reorient (rotate) the plot by using the arrow keys in the obvious way to rotate left, right, up or down. With the plot visible, the user presses the Esc (escape) key and chooses a particular rotation or the proceed option which produces the next plot in the sequence. These rotations may be used

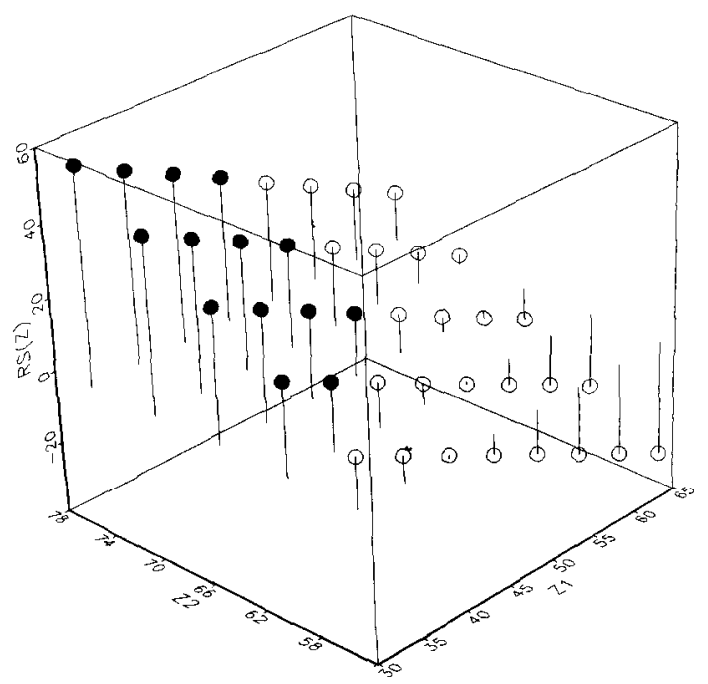

Fig. 10. Simultaneous region of significance for combinations of age and initial MRUS values. 


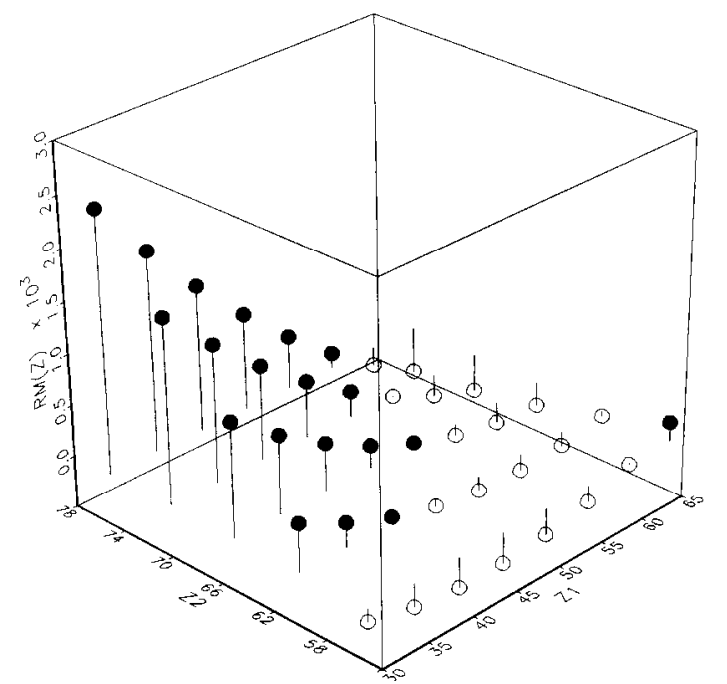

Fig. 11. Marginal region of significance for combinations of age and initial MRUS values.

more than once and in combination. The result of rotating twice to the right is shown in Fig. 6. Note that the $\boldsymbol{Z}_{1}$ and $\boldsymbol{Z}_{2}$ axes are positioned differently than in Fig. 5.

In Fig. 7, the values of $\mathrm{D}(\mathrm{Z})$ are shown in a different form. Lines emanating from the zero plane

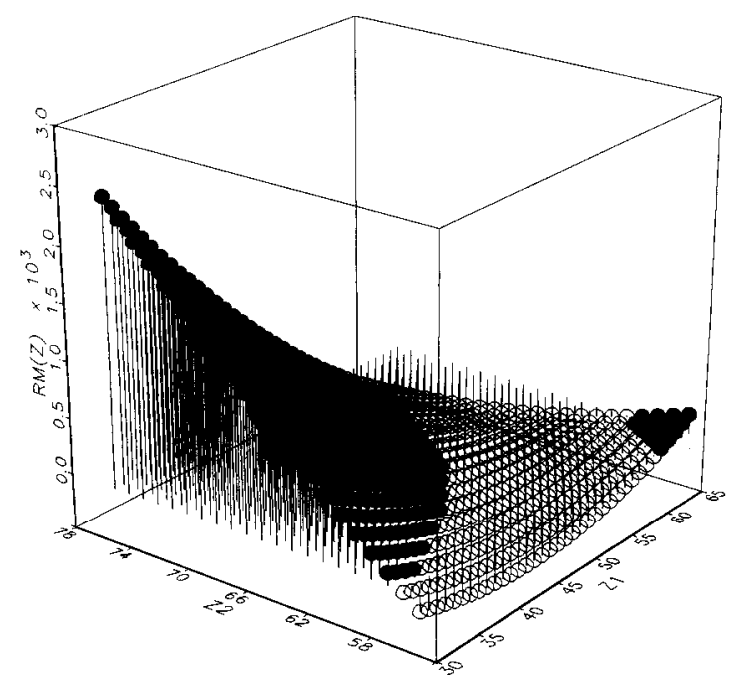

Fig. 12. Marginal region of significance for combinations of age and initial MRUS values when more $\mathrm{Z}$ values are selected for plotting (cf Fig. 11).

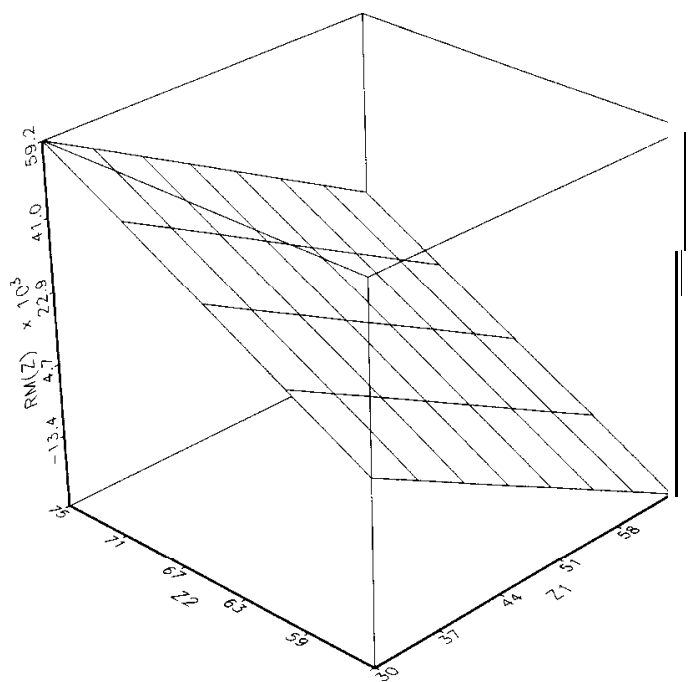

Fig. 13. Surface plot of $D(Z)$ for combinations of age and initial MRUS values.

indicate the value of $D(Z)$ by circles: solid circles are positive values; open circles, negative. This provides the same information as does Fig. 6: it is offered as an alternative which may be preferred by some users. Since the plots shown are produced sequentially in our program, and since we did not

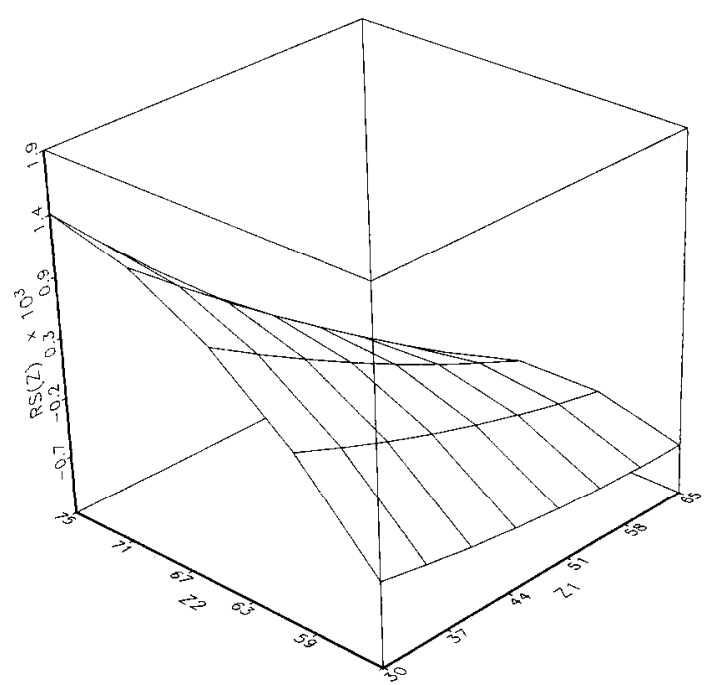

Fig. 14. Surface plot of simultaneous region of significance for combinations of age and initial MRUS values. 
reorient the axes after printing Fig. 6, the axes in Fig. 7 are the same as those in Fig. 6. We can, however, get back to the original orientation of Fig. 5 by rotating Fig. 7 as shown in Fig. 8. Note that the appearance of these plots depends on the choices made for the range of values of $\boldsymbol{Z}_{\mathbf{1}}$ and $\boldsymbol{Z}_{\mathbf{2}}$ and, in particular, on the increment between them. Figs. 7 and 8 are based on the choices $Z_{1}=30(5) 65$ and $Z_{2}=55(5) 75$, as indicated earlier. Had we chosen instead $Z_{1}=30(1) 65$ and $Z_{2}=55(1) 75$, Fig. 7 would appear as shown in Fig. 9. It is seen that the points at which $D(Z)$ is evaluated in Fig. 9 are much more dense than in Fig. 7. Which is preferred is a matter of personal preference and/or the purpose of the investigation. The user may have to experiment with various choices to find the optimal density of points in particular situations.

Plots of the type shown above are also produced representing the simultaneous and marginal regions of significance as shown in Figs. 10 and 11, respectively. The regions where significant differences exist correspond to those values of $Z_{1}$ and $\boldsymbol{Z}_{2}$ where the circles are solid (and on top of the line emanating from zero). Note again that the simultaneous region of significance is smaller than the marginal. Also, comparing Figs. 10 and 11 with Fig. 8, shows clearly the difference between $\mathrm{D}(\mathrm{Z})$ and the functions $\mathrm{R}(\mathrm{Z})$. The $\mathrm{D}(\mathrm{Z})$ plot (Fig. 8) shows where $D(Z)$ is positive; the plots of $R(Z)$ show whether or not $\mathrm{D}(\mathrm{Z})$ is significantly different from zero. Finally, note the (marginally) significant point in the far comer of Fig. 11 corresponding to $Z_{1}=65$ and $Z_{2}=75$. While this can happen, one should note that this point corresponds to the 2 most extreme values of $Z_{1}$ and $Z_{2}$. The user is free to choose any ranges for $\boldsymbol{Z}_{1}$ and $Z_{2}$, but should, in general, be careful to limit this choice to regions where data points exist.

As another example of the ways in which the choice of the increments between the $\mathrm{Z}$ values influences the appearance of the plot, we offer Fig. 12 which is to Fig. 11 as Fig. 9 is to Fig. 8.

Other plots produced by our program include surface plots of $\mathbf{D}(\mathbf{Z})$ and the regions of significance. Examples are shown in Figs. 13 and 14. The surface plot of $D(Z)$ is entirely similar to Fig. 5, except that a plane has been fit to the data in Fig. 13. For $\mathrm{Q}=2, \mathrm{D}(\mathrm{Z})$ will always be a plane and this correspondence will be true. $\mathrm{R}(\mathrm{Z})$, on the other hand, is a true surface as is seen in Fig. 14. Again, this surface can be made smoother by selecting more $\mathrm{Z}$ values to be plotted.

\section{Acknowledgement}

Supported by DE 08730 from the National Institute for Dental Research.

\section{Appendix}

A full set of PC programs for estimating treatment effects and/or performing specialized analyses in the context of simple linear regression, a set which includes this program and others cited in the reference list, can be obtained on 5.25 " or 3.5 " diskettes (please request type) by sending $\$ 25$ to defray the cost of handling and licensing fees. These programs require a 80386- or 80486-based personal computer (PC) running the MS-DOS operating system (version 5.0 or higher is recommended, although versions as low as 3.3 will suffice). 80386 computers must also be equipped with a 80387 math coprocessor. At least 4 MB of memory are required, and must be available to GAUSS386i, i.e., not in use by memory resident programs such as Windows. EGA or VGA graphic capabilities are required to display the color graphics; VGA or SVGA is suggested to display optimally the graphic results. Runtime modules are supplied with the programs so that no additional software (i.e., compiler or interpreter) is required to run these programs. One can create and edit ASCII data sets for use by these programs using the full screen editor supplied with MS-DOS version 5.0. The programs are written and compiled using GAUSS386i, version 3.0, require no additional installation or modification, and are run with a single command. When requesting the programs, address inquiries to the corresponding author and make checks payable to Baylor College of Dentistry.

\section{R eferences}

[1] Kowalski CJ, Schneiderman ED and Willis SM: Assessing the effect of a treatment when subjects are growing at different rates.Int J Biomed Comput, 37 ( 1994) 151 - 159. 
[2] Johnson PO and Neyman J: Tests of certain linear hypotheses and their application to some educational problems, Stat Res Mem, 1 (1936) 57-93.

[3] Johnson PO and Fay LC: The Johnson-Neyman technique, its theory and application, Psychometrika, 15 (1950) 349-367.

[4] Potthoff RF: On the Johnson-Neyman technique and some extensions thereof, Psychometrika, 29 (1964) 241-256.

[5] Potthoff RF: Johnson-Neyman technique. In: Encyclopedia of Statistical Sciences, vol. 4 (Eds: SKotz and NL Johnson), Wiley, New York, 1983.

[6] Rikkers LF, Rudmon D, Galambos JT, Fulenwider JT, Milliken WJ, Kutner MH, Smith RB, Salam AA, Jones
PJ and Warren WD: A randomized controlled trial of the distal splenorenal shunt,Ann Surg, 188 (1978) 271-282.

[7] Brogan DR and Kutner MH: Comparative analyses of pretest-posttest research designs, Am Stat, 34 (1980) 229-232.

[8] Ten Have TR, Kowalski CJ and Schneiderman ED: PC program for analyzing one-sample longitudinal data sets which satisfy the two-stage polynomial growth curve model, Am J Hum Biol, 3 (1991) 269-279.

[9] Ten Have TR, Kowalski CJ, Schneiderman ED and Willis SM: A PC program for performing multigroup longitudinal comparisons using the Potthoff-Roy analysis and orthogonal polynomials, Int J Biomed Comput, 30 (1992) 103-112. 